\title{
ON THE LATTICE OF VARIETIES OF COMPLETELY REGULAR SEMIGROUPS
}

\author{
P. R. JONES \\ (Received 15 September 1982) \\ Communicated by T. E. Hall
}

\begin{abstract}
Several morphisms of this lattice $\widetilde{V}(\mathbf{C R})$ are found, leading to decompositions of it, and various sublattices, into subdirect products of interval sublattices. For example the map $\mathbf{V} \rightarrow \mathbf{V} \cap \mathbf{G}$ (where $\mathbf{G}$ is the variety of groups) is shown to be a retraction of $\mathcal{V}(\mathbf{C R})$; from modularity of the lattice $\mathcal{T}(\mathbf{B G})$ of varieties of bands of groups it follows that the map $\mathbf{V} \rightarrow(\mathbf{V} \cap \mathbf{G}, \mathbf{V} \vee \mathbf{G})$ is an isomorphism of $(\mathbf{B G})$.

In addition, identities are provided for the varieties of central completely regular semigroups and of central bands of groups, answering questions of Petrich.
\end{abstract}

1980 Mathematics subject classification (Amer. Math. Soc.): $20 \mathrm{M} 07$.

\section{Introduction}

The class CR of completely regular semigroups (also called unions of groups) forms a variety of universal algebras when considered as semigroups with the additional unary operation $x \rightarrow x^{-1}$. Particular sublattices of the lattice $\mathscr{V}(\mathbf{C R})$ of varieties of completely regular semigroups have been the subject of intense study: for instance the lattice $\mathcal{V}(\mathbf{G})$ of varieties of groups (see [10]), the lattice $\mathcal{V}(\mathbf{C S})$ of varieties of completely simple semigroups $[8,9,13-17]$ and the lattice $\mathcal{T}(\mathbf{B})$ of varieties of bands [1,2,3].

It is the main aim of this paper to study various morphisms of the lattice $\mathfrak{T}(\mathbf{C R})$, of the type used in [6] and [13], to extend the knowledge of these special sublattices to larger sublattices by means of subdirect decompositions. In [6], Hall and the author showed that the map $\mathbf{V} \rightarrow(\mathbf{V} \cap \mathbf{B}, \mathbf{V} \vee \mathbf{B})$ is an isomorphism of

(C) 1983 Australian Mathematical Society $0263-6115 / 83 \$ A 2.00+0.00$ 
the lattice $\mathcal{V}(\mathbf{B G})$ of varieties of bands of groups upon a subdirect product of the interval sublattices $\mathcal{V}(\mathbf{B})$ and [B, BG]; from this it could be deduced that $\mathscr{V}(\mathbf{B G})$ is modular, a powerful result some of whose implications are treated here. In [13] a similar decomposition of $\mathcal{V}(\mathbf{C S})$ using $\mathbf{G}$ instead of $\mathbf{B}$ was given. The varieties $\mathbf{B}$ and $\mathbf{G}$ are called neutral ([4]) (in their respective lattices).

We show (Section 3) that $\mathbf{G}$ is in fact neutral in $\mathcal{V}(\mathbf{B G})$, and also (Section 4) in the lattice $\mathcal{V}(\mathbf{O C R})$ of orthodox completely regular semigroups; a partial result is obtained in $\mathscr{V}(\mathbf{C R})$ itself. Our technique has the advantage that explicit knowledge of the (relatively) free objects in the relevant varieties is not required.

Among the similar results obtained it is shown that $\mathbf{C S}$ is neutral in $\mathscr{V}(\mathbf{B G})$ and that every variety of normal bands is neutral in the whole lattice $\mathcal{T}(\mathbf{C R})$.

In [16] Petrich and Reilly proved a result of a similar type, to the effect that the variety CCS of central completely simple semigroups is neutral in the lattice $\mathcal{V}(\mathbf{C S})$. It would be of interest to know to what extent that result could be generalized.

In the final section two questions posed by Petrich in [12] are answered: identities are provided for the varieties CCR and CBG of central completely regular semigroups and bands of groups respectively.

\section{Preliminaries}

In general, for semigroup theoretic notation and terminology we follow Howie [7]. However we make the following conventions: the term "completely regular" will be abbreviated to "c.r." throughout; for any element $x$ of a c.r. semigroup, $x^{-1}$ and $x^{0}$ will denote respectively the inverse of $x$ in, and the identity of, the maximal subgroup to which it belongs; thus $x^{0}=x x^{-1}=x^{-1} x$.

For convenience we present a list of the abbreviations used for various varieties of c.r. semigroups:

$$
\begin{aligned}
\mathbf{C R} & =\text { c.r. semigroups } \\
\mathbf{C S} & =\text { completely simple semigroups, } \\
\mathbf{L Z}[\mathbf{R Z}] & =\text { left [right] zero semigroups } \\
\mathbf{R B} & =\text { rectangular bands, } \\
\mathbf{B} & =\text { bands } \\
\mathbf{G} & =\text { groups } \\
\mathbf{B G} & =\text { bands of groups } \\
\mathbf{N B} & =\text { normal band } \\
\mathbf{N B G} & =\text { normal bands of groups }
\end{aligned}
$$




$$
\begin{aligned}
\mathbf{S L} & =\text { semilattices, } \\
\mathbf{S L G} & =\text { semilattices of groups, } \\
\mathbf{T} & =\text { trivial semigroups. }
\end{aligned}
$$

Further, prefixing $\mathbf{O}$ to any variety $\mathbf{V}$ will indicate the subvariety of $\mathbf{V}$ consisting of those orthodox members of $\mathbf{V}$ : thus $\mathbf{O V}=\mathbf{V} \cap \mathbf{O C R}$. Prefixing $\mathbf{C}$ to $\mathbf{V}$ will indicate the subvariety of $\mathbf{V}$ consisting of those central members of $\mathbf{V}$ : thus $\mathbf{C V}=\mathbf{V} \cap \mathbf{C C R}$. (A c.r. semigroup is central if the product of any two of its idempotents lies in the centre of the maximal subgroup to which it belongs.) That CV is indeed a variety will follow from Theorem 5.1, where it is shown that CCR is itself a variety.

For identities defining these and various other varieties of c.r. semigroups we refer the reader to [12].

For reference we quote here the following important result mentioned in the introduction.

Result 2.1 [6, Theorem 3.1]. The lattice $\mathfrak{V}(\mathbf{B G})$ is modular.

Its importance in the context of this paper stems from the next result, for which some preparation is required. In general, for lattice theoretic notation and terminology we follow Grätzer [4].

An element of a lattice $L$ is called neutral ([4], Section III.2) if for all $a, b$, in $L$,

(i) $(a \vee b) \wedge d=(a \wedge d) \vee(b \wedge d)$,

(ii) $(a \wedge b) \vee d=(a \vee d) \wedge(b \vee d)$, and

(iii) $a \wedge d=b \wedge d$ and $a \vee d=b \vee d$ together imply $a=b$.

Clearly $d$ satisfies (i) if and only if the map $a \rightarrow a \wedge d$ is a retraction of $L$ upon the principal ideal ( $a$ ] generated by $a$; a dual statement is valid for (ii). Thus $d$ is neutral if and only if the map $a \rightarrow(a \wedge d, a \vee d)$ is an isomorphism upon a subdirect product of $(a]$ and its dual $[a)$.

Specializing Theorem III.2.6 of [4] we obtain

RESULr 2.2. In a modular lattice any element satisfying either (i) or (ii) is neutral.

\section{Some morphisms in $\checkmark(\mathbf{C R})$}

The first main result of this section, which generalizes Theorem 4.4 of [13], is the following.

THEOREM 3.1. The mapping $\mathbf{V} \rightarrow \mathbf{V} \cap \mathbf{G}$ is a retraction of $\mathcal{T}(\mathbf{C R})$ upon $\mathcal{V}(\mathbf{G})$. 
Proof. Let $U, V \in \mathcal{V}(\mathbf{C R})$. The inclusion

$$
(\mathbf{U} \cap \mathbf{G}) \vee(\mathbf{V} \cap \mathbf{G}) \subseteq(\mathbf{U} \vee \mathbf{V}) \cap \mathbf{G}
$$

is clear. To prove this converse let $G$ be a group belonging to $\mathbf{U} \vee \mathbf{V}$. Thus there exist c.r. semigroups $A \in \mathbf{U}$ and $B \in \mathrm{V}$, a (regular) subdirect product $T$ of $A$ and $B$ and a morphism $\phi$ of $T$ upon $G$. We will show that every finitely generated subgroup of $G$ belongs to $(\mathbf{U} \cap \mathbf{G}) \vee(\mathbf{V} \cap \mathbf{G})$, so that $G$ itself does.

So let $F$ be such a subgroup, generated by $\left\{g_{1}, \ldots, g_{n}\right\}$, say. For each $i$, let $y_{i} \in T$ be such that $y_{i} \phi=g_{i}$, and let $e_{i}=y_{i}^{0}$. Put $e=\left(e_{1} \cdots e_{n}\right)^{0}$. Since $T$ is c.r., each $e y_{i} e \mathcal{H} e$. Thus $\left\{e y_{1} e, \ldots, e y_{n} e\right\}$ generates a subgroup $H$, say, of $H_{e}$. Clearly $\left(e y_{i} e\right) \phi=g_{i}$, since $G$ is a group, so $H \phi=F$.

Now since $H$ is a subgroup of $A \times B, e=(a, b)$ for some idempotents $a \in A$, $b \in B$. But for any $(u, v) \in T,(u, v) \mathcal{H C}(a, b)$ if and only if $u \mathcal{H C} a$ (in $A)$ and $v \mathcal{H C} b$ (in $B$ ), so $H_{e}$ is isomorphic to a subgroup of $H_{a} \times H_{b}$. Since $H_{a} \in \mathbf{U} \cap \mathbf{G}$ and $H_{b} \in \mathbf{V} \cap \mathbf{G}, H_{e}, H$ and $F$ in turn belong to $(\mathbf{U} \cap \mathbf{G}) \vee(\mathbf{V} \cap \mathbf{G})$, as required.

Applying Results 2.1 and 2.2 to this theorem immediately yields the following, the final statement of which is Theorem 5.5 of [13].

COROLlaRY 3.2. The variety $\mathbf{G}$ is neutral in $\widetilde{V}(\mathbf{B G})$, that is, the map $\mathbf{V} \rightarrow(\mathbf{V} \cap$ $\mathbf{G}, \mathbf{V} \vee \mathbf{G})$ is an isomorphism of $\mathscr{V}(\mathbf{B G})$ upon a subdirect product of $\mathscr{V}(\mathbf{G})$ with the interval [G, BG]. In particular $\mathbf{G}$ is neutral in $\mathcal{V}(\mathbf{C S})$.

We do not know whether $\mathbf{G}$ is neutral in $\mathcal{V}(\mathbf{C R})$. (See, however, Theorem 4.1.)

The proof of Theorem 3.1 may be easily modified (essentially by replacing $\mathcal{H}$ by $\mathcal{D}$ throughout) to obtain

THEOREM 3.3. The map $\mathbf{V} \rightarrow \mathbf{V} \cap \mathbf{C S}$ is a retraction of $\mathscr{V}(\mathbf{C R})$ upon $\mathscr{V}(\mathbf{C S})$.

Corollary 3.4. The variety $\mathbf{C S}$ is neutral in $\Upsilon$ (BG).

In [6], Proposition 3.5 it was shown that SL is neutral in the entire lattice $\mathcal{V}(\mathbf{C R})$. We use a similar approach to prove

THEOREM 3.5. The variety $\mathbf{L Z}$ is neutral in $\mathfrak{V}(\mathbf{C R})$.

Proof. We show directly that the map

$$
\mathbf{V} \rightarrow(\mathbf{V} \cap \mathbf{L Z}, \mathbf{V} \vee \mathbf{L Z})
$$


is an order isomorphism. It is clearly order preserving. So suppose $\mathbf{U}, \mathbf{V}$ in $\mathfrak{V}(\mathbf{C R})$ are such that

$$
\mathbf{U} \cap \mathbf{L Z} \subseteq \mathbf{V} \cap \mathbf{L Z} \text { and } \mathbf{U} \vee \mathbf{L Z} \subseteq \mathbf{V} \vee \mathbf{L Z}
$$

we must show $\mathbf{U} \subseteq \mathbf{V}$.

Note that since $\mathbf{L Z}$ is an atom of the lattice $\mathscr{V}(\mathbf{C R})$ either $\mathbf{L Z} \subseteq \mathbf{V}$ or $\mathbf{V} \cap \mathbf{L Z}=\mathbf{T}$. In the former case the second inclusion yields $\mathbf{U} \subseteq \mathbf{V}$ immediately, so from now on assume $\mathbf{V} \cap \mathbf{L Z}=\mathbf{T}$. In that case $\mathbf{U} \cap \mathbf{L Z}=\mathbf{T}$ also, so both $\mathbf{U}$ and $\mathbf{V}$ consist entirely of semilattices of right groups.

Now let $S \in \mathbf{U}$. Thus $S \in \mathbf{V} \vee \mathbf{L Z}$ and there exist $A \in \mathbf{V}$ and $L \in \mathbf{L Z}$, a subdirect product $T$ of $A$ and $L$ and a morphism $\phi$ of $T$ upon $S$. For each element $a$ of $A$ define $a \bar{\phi}=(a, l) \phi$, for some $(a, l) \in T$. Suppose $(a, l)$ and $(a, m) \in T$ : since $L$ is a left zero semigroup it follows that $l \mathrm{~L} m$, whence $(a, l) \mathscr{L}(a, m)$ in $T$ and $(a, l) \phi \mathcal{L}(a, m) \phi$ in $S$. But the $\mathcal{D}$-class of $S$ containing $(a, l) \phi$ is a right group, so $(a, l) \phi \mathcal{H}(a, m) \phi$. Now $\left(a^{0}, l\right)$ is the identity of the $\mathcal{G}$-class of $(a, l)$ in $T$, so $\left(a^{0}, l\right) \phi$ is the identity of the $\mathcal{G}$-class of $(a, l) \phi$ in $S$. Therefore

$$
(a, l) \phi=\left(\left(a^{0}, l\right)(a, m)\right) \phi=\left(a^{0}, l\right) \phi(a, m) \phi=(a, m) \phi .
$$

Thus $\bar{\phi}$ defines a mapping of $A$ into $S$ which is clearly a surjective morphism. Hence $S \in \mathrm{V}$, as required.

From duality it follows that $\mathbf{R Z}$ is also neutral in $\mathscr{V}(\mathbf{C R})$. From the definition of neutrality it is easily seen that the neutral elements of any lattice form a sublattice. Thus RB is neutral and in fact the sublattice of $\mathcal{T}(\mathbf{C R})$ generated by $\mathbf{L Z}, \mathbf{R Z}$ and SL consists of neutral elements. This sublattice is precisely the lattice $(\mathbf{N B})$ (see, for example, [7, page 124]), giving

COROLlaRY 3.6. Every variety of normal bounds is neutral in $\mathscr{T}(\mathbf{C R})$.

Specializing to $\mathfrak{V}(\mathbf{B G})$ and noting, additionally, neutrality of $\mathbf{B}$ there (implicit in [6], Proposition 3.4) it follows that the sublattice generated by LZ, RZ, SL, G, CS and B consists of neutral elements. For a diagram of the bulk of this sublattice see Diagram 1 of [11] (and for the "missing join" see [6]). In particular, for instance, NBG and OBG are neutral in $\mathcal{T}(\mathbf{B G})$.

\section{Neutrality of $G$ in OCR}

Before proving the main result of this section we remind the reader of some facts concerning $\mu$, the greatest idempotent separating congruence on a regular semigroup. We will make use of the fact that on any orthodox semigroup $S$ the 
intersection $\mu \cap \gamma$ is trivial, $\gamma$ denoting the least inverse semigroup congruence on $S$. (See, for instance [7], Section VI.4.) Thus $S$ is isomorphic to a subdirect product of $S / \mu$ and $S / \gamma$. When $S$ is, further, c.r., $S / \gamma$ is clearly a semilattice of groups. We also make use of the fact that if $\phi: S \rightarrow T$ is a surjective morphism of regular semigroups, and if $a \mu b$ in $S$, then $a \phi \mu b \phi$ in $T$.

\section{THeOREM 4.1. The variety $\mathbf{G}$ is neutral in $\mathbf{O C R}$.}

Proof. We again prove directly that the map $\mathbf{U} \rightarrow(\mathbf{U} \cap \mathbf{G}, \mathbf{U} \vee \mathbf{G})$ is an order isomorphism. So let $U, V \in \mathcal{V}(\mathbf{O C R})$ and suppose

$$
\mathbf{U} \cap \mathbf{G} \subseteq \mathbf{V} \cap \mathbf{G} \text { and } \mathbf{U} \vee \mathbf{G} \subseteq \mathbf{V} \vee \mathbf{G} .
$$

Observe first that since, by Theorem 3.3, the map A $\rightarrow \mathbf{A} \cap \mathbf{C S}$ is a morphism of $\Upsilon(\mathbf{C R})$, the above inequalities yield

$$
(\mathbf{U} \cap \mathbf{C S}) \cap \mathbf{G} \subseteq(\mathbf{V} \cap \mathbf{C S}) \cap \mathbf{G} \text { and }(\mathbf{U} \cap \mathbf{C S}) \vee \mathbf{G} \subseteq(\mathbf{V} \cap \mathbf{C S}) \vee \mathbf{G} \text {. }
$$

By Corollary 3.2, $\mathbf{G}$ is neutral in $\widetilde{V}(\mathbf{C S})$, so $\mathbf{U} \cap \mathbf{C S} \subseteq \mathbf{V} \cap \mathbf{C S}$. Now if $\mathbf{U}$ does not contain $\mathbf{S L}$ then $\mathbf{U} \subseteq \mathbf{C S}$, giving $\mathbf{U} \subseteq \mathbf{V}$. Similarly if $\mathbf{V}$ does not contain $\mathbf{S L}$, $\mathbf{V} \subseteq \mathbf{C S}$ and $\mathbf{U} \subseteq \mathbf{U} \vee \mathbf{G} \subseteq \mathbf{V} \vee \mathbf{G} \subseteq \mathbf{C S}$, giving $\mathbf{U} \subseteq \mathbf{V}$ again.

From now on, then, we assume both $\mathbf{U}$ and $\mathbf{V}$ contain $\mathbf{S L}$. Let $\mathbf{S} \in \mathbf{U}$. Then $S \in \mathbf{V} \vee \mathbf{G}$ and there exist $A \in \mathbf{V}$ and $G \in \mathbf{G}$, a subdirect product $T$ of $A$ and $G$ and a morphism $\phi$ of $T$ upon $S$. Let $a \in A$ and suppose $(a, g)$ and $(a, h) \in T$. Then $(a, g) \mu(a, h)$ and so $(a, g) \phi \mu(a, h) \phi$ in $S$. Hence $(a, g) \phi \mu^{\natural}=(a, h) \phi \mu^{\natural}$ in $S / \mu$ ( $\mu^{\natural}$ denoting the natural map). The map $\theta: A \rightarrow S / \mu$ given by

$$
a \theta=(a, g) \phi \mu^{\natural} \quad \text { for some }(a, g) \in T,
$$

is therefore well defined and is clearly a surjective morphism. Therefore $S / \mu \in \mathbf{V}$.

Clearly $S / \gamma \in \mathbf{U} \cap \mathbf{S L G}=(\mathbf{U} \vee \mathbf{S L}) \cap(\mathbf{G} \vee \mathbf{S L})=(\mathbf{U} \cap \mathbf{G}) \vee \mathbf{S L}$, since SL $\subseteq \mathbf{U}$ and SL is neutral in $\mathcal{V}(\mathbf{C R})$ (see Section 3). Therefore $S / \gamma \in(\mathbf{V} \cap \mathbf{G}) \vee \mathbf{S L}$ $\subseteq \mathbf{V}$. Since $S$ is isomorphic to a subdirect product of $S / \mu$ and $S / \gamma, S \in \mathbf{V}$ also.

\section{Central c.r. semigroups}

Petrich posed the following two problems (among others) in Section 7 of [12]. Problem 4. Is CBG defined by the identity

$$
a^{0} b^{0} a=a b^{0} a^{0} \text { ? }
$$

Problem 6. Is CCR a variety? If so find identities defining it.

In the theorem below we answer each question in the affirmative by providing a single identity for CCR which reduces to (1) in bands of groups. We will make use 
of the fact ([14], Proposition 6.2; see also [12], Lemma 3.5) that CCS is defined, within $\mathrm{CS}$, by (1).

THEOREM 5.1. a) The class CCR is defined by the identity

$$
\left(a^{0} b^{0} a\right)\left(b^{0} a^{0}\right)^{0}=\left(a^{0} b^{0}\right)^{0}\left(a b^{0} a^{0}\right),
$$

and is therefore a variety. In fact $\mathbf{C C R}$ consists precisely of the semilattices of central completely simple semigroups.

b) The variety CBG is defined by the identity (1).

Proof. a) First let $S \in \mathbf{C C R}$, and let $a, b \in S$. Using Lemma 1 of [5],

$$
\begin{aligned}
a^{0} b^{0} a & =\left[\left(a^{0} b^{0} a\right)\left(a^{0} b^{0} a\right)^{-1} a^{0}\right]\left[b^{0} a\left(a^{0} b^{0} a\right)^{-1} a^{0} b^{0}\right]\left[a\left(a^{0} b^{0} a\right)^{-1}\left(a^{0} b^{0} a\right)\right] \\
& =\left[\left(a^{0} b^{0} a\right)^{-1}\left(a^{0} b^{0} a\right) a^{0}\right]\left[b^{0} a\left(a^{0} b^{0} a\right)^{-1} a^{0} b^{0}\right]\left[a\left(a^{0} b^{0} a\right)^{0}\right] \\
& =\left[\left(a^{0} b^{0} a\right)^{0}\right]\left[b^{0} a\left(a^{0} b^{0} a\right)^{-1} a^{0} b^{0}\right]\left[\left(a^{0} b^{0}\right)^{0} a\left(a^{0} b^{0} a\right)^{0}\right] \\
& =x y z, \text { say, }
\end{aligned}
$$

where each of these terms belongs to the same $\mathscr{D}$-class of $S$, the middle term $y$ is idempotent, and $z \mathscr{K} x$ (since $z \mathscr{R} a^{0} b^{0} \Re x$ and $z \mathscr{L} a^{0} b^{0} a \mathscr{C} x$ ). So in fact $a^{0} b^{0} a=$ $z^{0} y^{0} z$.

From the definition of centrality it is clear that each $\mathscr{D}$-class of $S$ is a central completely simple semigroup and therefore satisfies (1), so that $z^{0} y^{0} z=z y^{0} z^{0}$. Thus

$$
\begin{aligned}
a^{0} b^{0} a & =\left[\left(a^{0} b^{0}\right)^{0} a\left(a^{0} b^{0} a\right)^{0}\right]\left[b^{0} a\left(a^{0} b^{0} a\right)^{-1} a^{0} b^{0}\right]\left[\left(a^{0} b^{0} a\right)^{0}\right] \\
& =\left[\left(a^{0} b^{0}\right)^{0} a\left(a^{0} b^{0} a\right)^{0}\right]\left[\left(a^{0} b^{0} a\right)\left(a^{0} b^{0} a\right)^{-1} a^{0} b^{0}\right]\left[\left(a^{0} b^{0} a\right)^{0}\right] \\
& =\left(a^{0} b^{0}\right)^{0} a\left(a^{0} b^{0} a\right)^{0} a^{0} b^{0}\left(a^{0} b^{0} a\right)^{0} \\
& =\left(a^{0} b^{0}\right)^{0} a\left(a^{0} b^{0} a\right)^{0}\left(a^{0} b^{0} a\right) a^{-1}\left(a^{0} b^{0} a\right)^{0} \\
& =\left(a^{0} b^{0}\right)^{0} a\left(a^{0} b^{0} a\right) a^{-1}\left(a^{0} b^{0} a\right)^{0} \\
& =\left(a^{0} b^{0}\right)^{0}\left(a b^{0} a^{0}\right)\left(a^{0} b^{0} a\right)^{0} .
\end{aligned}
$$

On the other hand,

$$
\begin{aligned}
\left(a^{0} b^{0} a\right)\left(b^{0} a^{0}\right)^{0} & =\left(a^{0} b^{0} a\right)\left(a^{0} b^{0} a^{0}\right)\left(b^{0} a^{0}\right)^{-1} \\
& =\left(a^{0} b^{0} a\right)\left(a^{0} b^{0} a^{0}\right)^{0}\left(a^{0} b^{0} a^{0}\right)\left(b^{0} a^{0}\right)^{-1} \\
& =\left(a^{0} b^{0} a\right)\left(a^{0} b^{0} a^{0}\right)^{0}\left(b^{0} a^{0}\right)^{0} \\
& =\left(a^{0} b^{0} a\right)\left(a^{0} b^{0} a^{0}\right)^{0}, \text { since } a^{0} b^{0} a^{0} \varrho b^{0} a^{0},
\end{aligned}
$$


and so

$$
\begin{aligned}
& =\left(a^{0} b^{0}\right)^{0}\left(a b^{0} a^{0}\right)\left(a^{0} b^{0} a\right)^{0}\left(a^{0} b^{0} a^{0}\right)^{0}, \quad \text { using }(3), \\
& =\left(a^{0} b^{0}\right)^{0}\left(a b^{0} a^{0}\right)\left(a^{0} b^{0} a^{0}\right)^{0}, \quad \text { since } a^{0} b^{0} a \Re a^{0} b^{0} a^{0}, \\
& =\left(a^{0} b^{0}\right)^{0}\left(a b^{0} a^{0}\right), \quad \text { since } a b^{0} a^{0} \varrho a^{0} b^{0} a^{0} .
\end{aligned}
$$

\section{So $S$ satisfies (2).}

Conversely let $S$ be a c.r. semigroup which satisfies (2), and let $D$ be a 2 -class of $S$. Since $\mathscr{K}$ is a congruence on $D$, for any $a, b \in D$ we have $\left(b^{0} a^{0}\right)^{0}=\left(b^{0} a\right)^{0}$ and $\left(a^{0} b^{0}\right)^{0}=\left(a b^{0}\right)^{0}$ so that $(2)$ reduces to (1) in $D$. Hence each $\mathcal{D}$-class is a central completely semigroup. Now if $e$ and $f$ are idempotents of $S$, then $e f=\left[(e f)^{0} e\right]\left[f(e f)^{0}\right]$, where each of these two terms is an idempotent of $D_{e f}$. Their product thus lies in the centre of the maximal containing subgroup and $S$ is itself central.

The final statement of a) is now clear from the above proof.

b) Since the bands of groups are precisely the c.r. semigroups on which $\mathscr{K}$ is a congruence, this follows as in the proof above.

\section{References}

[1] A. P. Birjukov, 'Varieties of idempotent semigroups,' Algebra i Logika 9 (1970), 255-273

[2] C. F. Fennemore, 'All varieties of bands,' Math. Nachr. 48 (1971), I: 237-252, II: 253-262.

[3] J. A. Gerhard, 'The lattice of equational classes of idempotent semigroups,' J. Algebra 15 (1970), 195-224.

[4] G. Gr̈tzer, General lattice theory (Birkhauser Verlag, Basel, 1978).

[5] T. E. Hall, 'On regular semigroups,' J. Algebra 24 (1973), 1-24.

[6] T. E. Hall and P. R. Jones, 'On the lattice of varieties of bands of groups,' Pacific J. Math. 91 (1980), 327-337.

[7] J. M. Howie, An introduction to semigroup theory (Academic Press, London, 1976).

[8] P. R. Jones, 'Completely simple semigroups: free products, free semigroups and varieties,' Proc. Roval Soc. Edinburgh A 88 (1981), 293-313.

[9] C. I. MasevickiI, 'On identities in varieties of completely simple semigroups over abelian groups,' Contemporary algebra, Leningrad (1978), pp. 81-89 (Russian).

[10] H. Neumann, Varieties of groups (Springer-Verlag, New York, 1967).

[11] M. Petrich, 'Certain varieties and quasivarieties of completely regular semigroups,' Canad. $J$. Math. 29 (1977), 1171-1197.

[12] M. Petrich, 'On the varieties of completely regular semigroups,' Semigroup Forum 25 (1982), $153-170$.

[13] M. Petrich and N. R. Reilly, 'Varieties of groups and of completely simple semigroups,' Bull. Austral. Math. Soc. 23 (1981), 339-359.

[14] M. Petrich and N. R. Reilly, 'Near varieties of idempotent generated completely simple semigroups.' Algebra Universalis, to appear.

[15] M. Petrich and N. R. Reilly, "All varieties of central completely simple semigroups,' Trans. Anter. Math. Soc., to appear. 
[16] M. Petrich and N. R. Reilly, "Certain homomorphisms of the lattice of varieties of completely simple semigroups,' J. A ustral. Math. Soc., to appear.

[17] V. V. Rasin, 'On the lattice of varieties of completely simple semigroups,' Semigroup Forum 17 (1979), 113-122.

Department of Mathematics, Statistics and Computer Science

Marquette University

Milwaukee, Wisconsin 53233

U.S.A. 